\title{
ADQUISICIÓN Y PROCESAMIENTO DE IMÁGENES MULTIESPECTRALES BIOLÓGICAS: UN CASO DE ESTUDIO EN TEJIDO CARDIOVASCULAR
}

\author{
ACQUISITION AND PROCESSING OF BIOLOGICAL MULTI-SPECTRAL \\ IMAGES: A CASE OF STUDY IN CARDIOVASCULAR TISSUE
}

\footnotetext{
Deivid Botina $^{1}$, July Galeano ${ }^{1}$, Sandra Pérez ${ }^{2,3}$, Johnson Gar

Grupo de Materiales y Energía-MatyEr-. Linea Electromedicina. Instituto Tecnológico
Colombia. E-mail: Deivid.johan.botina.monsalve@gmail.com, julygaleano@itm.edu.co

2 Grupo de óptica y Espectroscopía - GOE

${ }^{3}$ Grupo de Investigación en Dinámica Cardiovascular Universidad Pontificia Bolivariana. Medellín-Colombia. E-mail: johnson.garzon@upb.edu.co, sandra.perezbu@upb.edu.co

Recibido: 3 Diciembre

Aceptado: 15 Diciembre

*Correspondencia del autor. Deivid Botina. Grupo de Materiales y Energía-MatyEr-. Linea Electromedicina. Instituto Tecnológico

Metropolitano. Medellín Colombia. E-mail: Deivid.johan.botina.monsalve@gmail.com, julygaleano@itm.edu.co
}

\begin{abstract}
RESUMEN
Este artículo presenta el uso de técnicas de adquisición y procesamiento imágenes multi-espectrales para el análisis de tejido biológico, específicamente cardiovascular. Se presentan diferentes tipos de arquitecturas, tales como los basados en filtros de interferencia y los que no requieren el uso de estos filtros. También se presentan ventajas y desventajas de ambos sistemas, así como técnicas para la calibración del color y fuentes de ruido. Además, se presentan dos tipos de técnicas de procesamiento de imágenes biológicas: análisis de componentes principales (PCA) y análisis de componentes independientes (ICA). Estas técnicas se han utilizado en el análisis de imágenes biológicas tales como piel humana y tejido gástrico. Sin embargo, y hasta nuestro conocimiento, dichas técnicas no han sido ampliamente utilizados en el análisis de tejido cardiovascular, de modo que se hace un enfoque especial en este tipo de tejido. Como resultado, se encontró que los métodos de PCA e ICA permiten la obtención de imágenes con una mejor definición de la morfología del tejido cardiovascular.
\end{abstract}

Palabras claves: tejido cardiovascular; análisis de componentes principales (PCA); análisis de componentes independientes (ICA)

\begin{abstract}
This article presents the use of multispectral acquisition and processing techniques in the analysis of biological tissue, specifically cardiovascular one. Different kinds of architectures are presented, like those based on interference filters and those that do not require the use of such as filters. Advantages and disadvantages of both systems are also presented, as well as techniques for color calibration and sources of noise. Also, two kinds of image processing techniques in biological images are presented: Principal Component Analysis (PCA) and Independent Component Analysis (ICA). Those techniques have been used in the analysis of biological images such as human skin, and colon. However, and up to our knowledge, those techniques have not been widely used in the analysis of cardiovascular tissue, so that special focus is done in this specific application. As a result, it is found that PCA and ICA methods allow obtaining images with a better definition of cardiovascular tissue morphology.
\end{abstract}

Key Words: cardiovascular tissue; principal component analysis (PCA); independent Component Analysis (ICA). 


\section{INTRODUCCIÓN}

Las imágenes multiespectrales están basadas en la adquisición de espectros de reflectancia por medio de dispositivos optoelectrónicos (1). Éste tipo de imágenes se están aplicando ampliamente en el estudio de tejidos biológicos, de allí que Jolivot, Vabres y Marzani desarrollaron un equipo para el estudio de enfermedades cutáneas y cáncer (2); Nakappan y su equipo implementaron una metodología de colposcopia en tiempo real para la detección del papiloma humano (3); Orfanoudaki y sus colaboradores emplearon imágenes multiespectrales para el estudio de biopsias de cuello uterino (4). También han resultado efectivas en la detección de úlceras y lesiones malignas en el sistema digestivo.

Actualmente las imágenes multiespectrales no son empleadas en el diagnostico clínico de patologías cardiovasculares, aunque existen investigaciones acerca del tema, no han logrado trascender del área académica, según estas es posible evidenciar un alto contraste entre los tejidos, posibilitando la obtención de nuevas imágenes del corazón o vasos sanguíneos (5) (6). Éste trabajo se desarrolla encaminado a lograr que las imágenes multiespectrales lleguen a satisfacer necesidades diagnósticas que las herramientas convencionales no alcanzan a cubrir.

\section{MATERIALES Y MÉTODOS}

\section{A. Materiales: sistemas de adquisición de imáge- nes multiespectrales}

1) Reflectancia de la luz: cuando una luz interactúa con un objeto pueden suceder tres cosas: la luz puede ser absorbida, reflejada y/o transmitida a través del objeto; todo depende del tipo de material y sus propiedades.

2) Sistemas de Adquisición de imágenes multiespectrales: un sistema multiespectral se compone de un hardware y un software. El conjunto de elementos que figuran en el hardware se presenta en la figura 1. El sistema consta de seis etapas que preferiblemente se deben disponer en un sitio estable para evitar alteraciones externas: A) Una fuente de luz encargada de dar la energía al sistema. En el caso de la figura 1, la luz blanca predispone los rangos visibles. B) Un sistema de lentes para colimar la luz que permite la disposición de una fuente de luz plana. C) Un fil- tro cuya función es dejar pasar solo la componente espectral con la cual se desea iluminar el objeto. D) Una muestra del objeto dispuesto para la toma de su respectiva imagen. E) Un sensor con la capacidad de capturar la imagen. F) Un computador para proceder al tratamiento de la imagen.

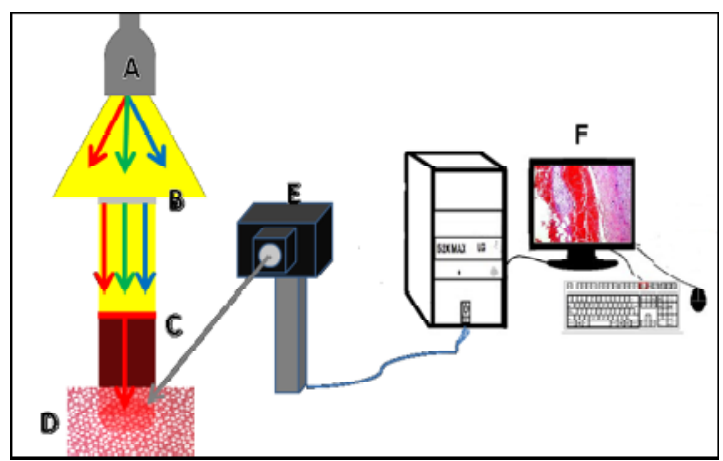

Figura 1. Sistema básico de adquisición de imágenes donde A) es la fuente de luz, B) El arreglo de lentes que coliman la luz, C) Filtro, D) Muestra, E) Cámara y F) Computador

Los filtros interferenciales son un elemento importante a la hora de montar un sistema multiespectral porque de estos filtros dependerán los resultados que se obtengan a la hora de tomar la imagen. Específicamente, los filtros permiten captar la imagen del objeto a una longitud de onda específica. Sin embargo existen otros dispositivos, diferentes a los filtros, capaces de variar las longitudes de onda de otras maneras como se verá más adelante. A continuación se presentan algunos ejemplos de sistemas de adquisición de imágenes que han sido utilizados en estudios científicos, cuya principal direncia entre el uno y el otro radica en el método de filtrado.

a) Mansouri, A., Marzani, F., Hardeberg, J., and Gouton, (7), utilizan un sistema de adquisición de imágenes espectrales muy similar al de la figura 1 , el único elemento que es reemplazado es el correspondiente al literal $\mathrm{C}$ por 9 filtros de fácil acceso que varían entre 400 y $1000 \mathrm{~nm}$, para abarcar toda la gama que el sensor de la cámara predispone. Cada uno de estos filtros ocupa una longitud de onda de aproximadamente $65 \mathrm{~nm}$ y su movimiento es controlado desde el computador.

b) Existe un sistema de adquisición de imágenes que se caracteriza por tener un filtro LCTF (Liquid crystal tunable filter) que sintoniza longitudes de onda según lo especifique su software; quiere decir que se controla por computador. Este filtro funciona gracias a 
que posee placas de cristal líquido conmutables. Existen filtros como estos que solo contienen los colores RGB, de allí en adelante existen otros que pueden llegar hasta sintonizar longitudes de onda en el lejano infrarrojo.Un ejemplo de un sistema de adquisición de imágenes multiespectrales donde se utiliza este filtro es el que fue implementado por J.Y. Hardeberg, et al (8), el cual al igual que en el ejemplo anterior solo se reemplaza el literal $\mathrm{C}$ de la figura 1 por el filtro LCTF sintonizable desde $400 \mathrm{~nm}$ hasta $720 \mathrm{~nm}$ vía electrónica por su interfaz RS-232.

c) "OL 490 Agile Light Source", es un artefacto capaz de sintonizar longitudes de onda desde $380 \mathrm{~nm}$ hasta $780 \mathrm{~nm}$ en el rango visible, y de 760nm hasta 1600 $\mathrm{nm}$ en el cercano infrarrojo, todo mediante un arreglo de micro espejos en su interior. El proceso que realiza al interior inicia desde una fuente lumínica de Xenón de $500 \mathrm{~W}$, la cual tiene su respectivo sistema de enfriamiento. Esta luz se colima para llegar a un difractor que divide la luz blanca en distintas longitudes de onda enfocándolas hacia el arreglo de micro espejos. Una vez en esta parte, dependiendo de la orden que se haya dado desde el computador, los espejos se alinean de tal manera que solo salga la luz con la longitud de onda seleccionada. Este resultado se enfoca nuevamente mediante unos lentes de modo que llegue hasta la guía de luz que mostrará la longitud de onda sintonizada en el exterior. La Figura 2 representa un diseño esquemático del interior del OL 490.

d) David T. D., et al (9) Realizaron un estudio en Landas donde deseaban diferenciar la piel sana de la piel con melanoma. Para esto se implementó un sistema de adquisición de imágenes hiperespectrales, donde el objeto encargado de dar las diferentes longitudes de onda fue un prisma dentro de un sistema de adquisición llamado PARISS (Prism and Reflector Imaging Spectroscopy System).

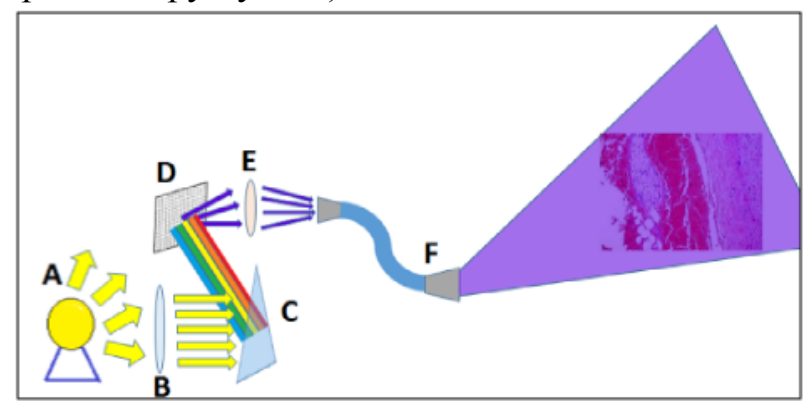

Figura 2. Estructura esquemática interna del OL 490 donde: A) Fuente lumínica de Xenón de 500W. B) Colimación de la luz C) Difracción de luz blanca. D) Arreglo de micro espejos. E) Focalización de luz filtrada. F) guía de luz.
Como se puede notar, en el rectángulo verde (figura 3), el sistema PARISS contiene una gran cantidad de elementos que hacen una adquisición espectral mucho más exacta. El sistema consiste en someter el tejido ante una luz halógena que se reflejará hacia el objetivo de microscopio. De allí pasará por el prisma con una longitud de onda específica, la luz pasará a un arreglo de espejos donde se hace llegar la imagen y la longitud de onda hasta el sensor CCD y hasta el espectrofotómetro. Esos datos se almacenan en una memoria interna de PARISS que se hace llegar hasta el computador para el tratamiento de imágenes. El software utilizado para este tratamiento y para la manipulación del sistema fue Zeiss Axioskop (Carl Zeiss Microimaging, Thornwood, NY). El sistema debe realizar un barrido por toda la imagen para concatenar la serie de imágenes tomadas, ya que estas son muy pequeñas para abarcar todo el tejido.

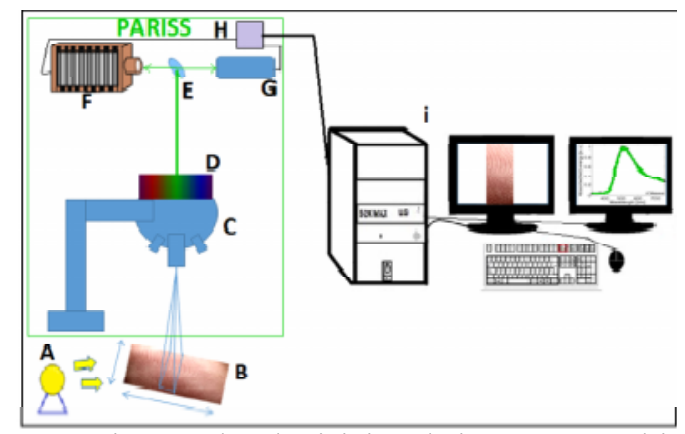

Figura 3. Sistema de adquisición de imágenes multiespectrales utilizado por David T. D., et al (9), donde: A) Fuente de luz, B) Muestra, C)Objetivo de microscopio, D) Prisma, E) Espejos internos, F) Cámara CCD, G) Espectrofotómetro, H) Módulo de Adquisición de datos PARISS, i) Computación encargada de controlar el sistema.

e) D. Duque y J. Garzón (10). utilizan un sistema en arquitectura confocal. La Figura 4 representa el esquema implementado por ellos.

El proceso realizado por este sistema inicia desde una fuente de luz que llega a un arreglo de lentes encargados de colimar la luz. Una vez el haz de luz es plano se hace llegar al Beam Spliter pasando por tres espejos que direccionan el haz. Finalmente la luz pasa por un lente de Fresnel (que asigna una longitud de onda específica), así se refleja la luz en el objetivo para llegar al espectrofotómetro.

\section{B. Métodos de procesamiento de imágenes: ICA y PCA}

1) Componentes del tejido cardiovascular: para realizar un análisis multiespectral del tejido cardio 


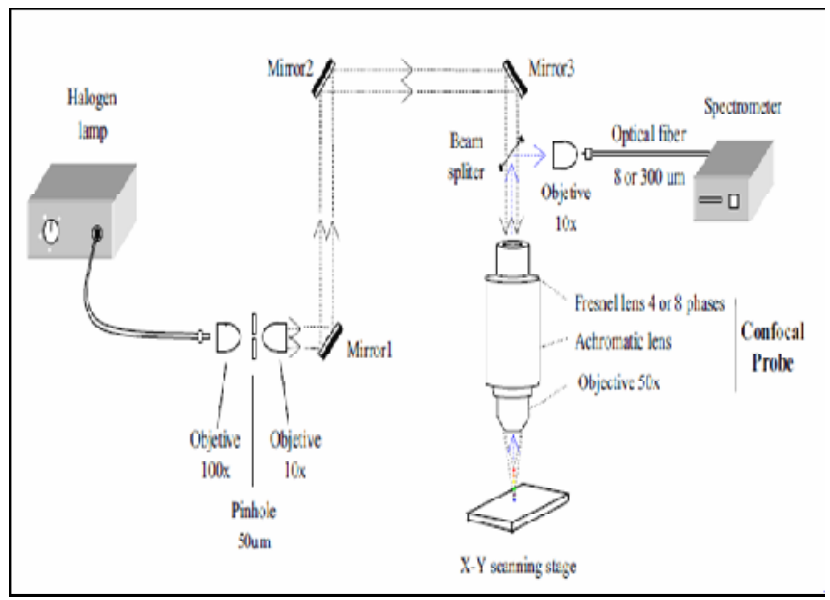

Figura 4. Sistema confocal implementado por D. Duque y J. Garzón donde la salida de la fuente de luz es colimada y dirigida por varios espejos para pasar por un beam spliter y al lente de Fresnel que dejará salir una longitud de onda que iluminará la muestra y llegará al espectrofotómetro para ser medida. Imagen tomada de D. Duque y J. Garzón (10).

vascular y conocer como la luz interactúa con este tejido, es necesario conocer las firmas espectrales de cada componente de éste. Entre los componentes encontramos la hemoglobina oxigenada, hemoglobina desoxigenada, metahemoglobina y Beta-caroteno. La figura 10 (11) contiene las firmas espectrales de dichos elementos. Esto con el fin de verificar que el cambio en las imágenes multiespectrales sea correcto al variar las longitudes de onda incidentes sobre el tejido. El uso de estas componentes será de utilidad al momento de utilizar los algoritmos ICA (Análisis de Componentes Independientes).

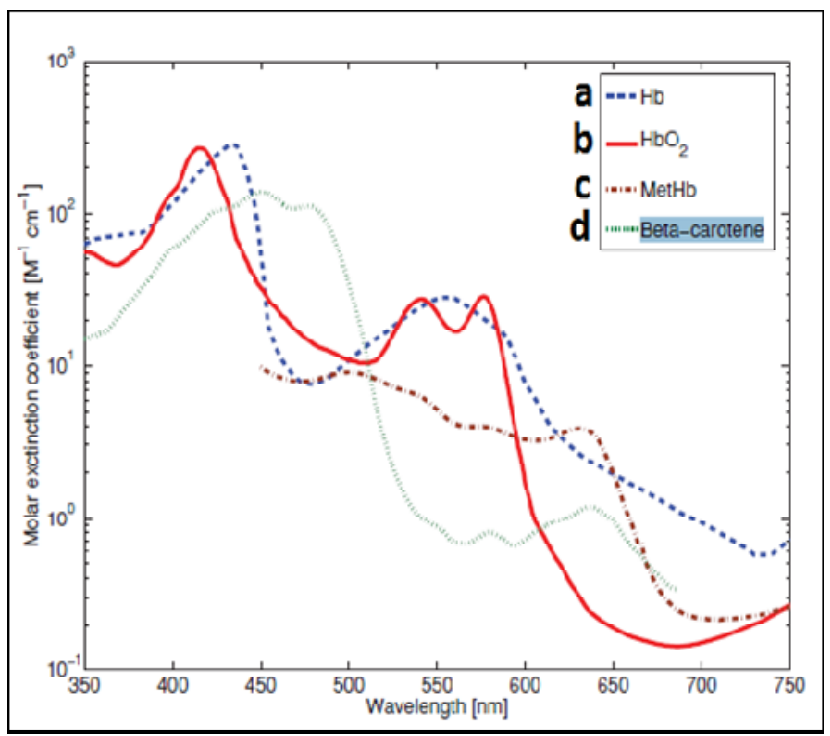

Figura 5. Coeficiente de extinción molar de: a) Hemoglobina desoxigenada, b) Hemoglobina oxigenada, c) Metahemoglobina y d) Beta-caroteno. Imagen tomada de Eivind et al (11).
2) Principal Components Analysis (PCA): PCA es una técnica que permite la descomposición de una señal o imagen en sus principales componentes. PCA está basado en un análisis estadístico, donde de una matriz de covarianza los eigenvalores y eigenvectores son calculados. De estos valores, los mayores corresponden a las principales componentes de la señal.

3)Análisis de Componentes Independientes (ICA): El método ideal para estimar un modelo ICA es la medida de no-gausianidad. El método clásico para medir no gausianidad es el método de Kurtosis. El algoritmo utilizado para ICA es el método Fast Ica. Dicho algoritmo requiere de un paso previo de "whitening" de la señal a descomponer. Luego, un método iterativo es implementado, el cual es presentado a continuación:

1. Selecciones un vector de valores aleatorios inicial w.

2. $\mathbf{w}+=E\{\mathbf{x} g(\mathbf{w} T \mathbf{x})\}-E\{g 0(\mathbf{w} T \mathbf{x})\} \mathbf{w}$

3. $\mathbf{w}=\mathbf{w}+/ \mathrm{kw}+\mathrm{k}$

4. si no hay convergencia regrese al paso 2 .

\section{RESULTADOS}

\section{A. Calibración espectral de sistemas de adquisi- cion de imágenes multiespectrales}

Se realizó la calibración de blancos tomando como patrón una muestra de teflón, de esta forma se ajustaron para cada longitud de onda los parámetros de apertura del diafragma y tiempo de exposición de la cámara. Obteniendo así las imágenes de la figura 6 .

Para la calibración del negro se capturó una imagen (figura 7) tapando completamente la lente de la cámara. Así se logró conocer el nivel basal de la cámara, es decir, el ruido intrínseco de la misma.

\section{B. Procesamiento de imágenes:}

Las muestras biológicas se tomaron de un corazón vacuno bajo medidas y cuidados específicos para la manipulación y desecho de material ex-vivo. 
Adquisición y procesamiento de imágenes multiespectrales biológicas. Botina. et al

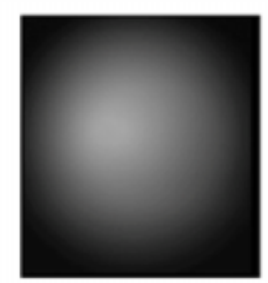

(a)

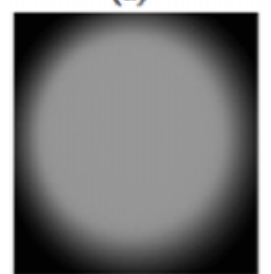

(c)

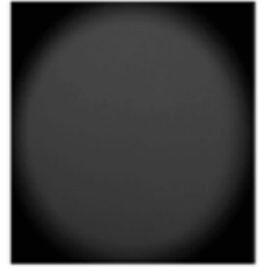

(e)

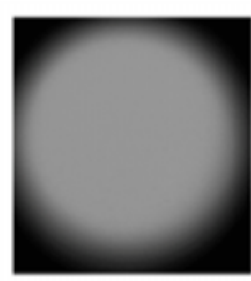

(b)

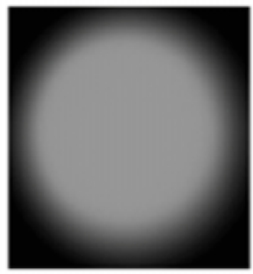

(d)

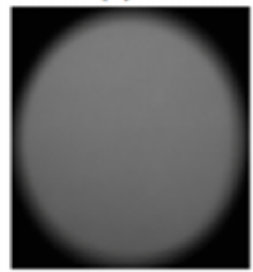

(f)
Figura 6. Imágenes de calibración de blancos. (a) $436 \mathrm{~nm}$. (b) $520 \mathrm{~nm}$. (c) $546 \mathrm{~nm}$. (d) $578 \mathrm{~nm}$. (e) $650 \mathrm{~nm}$. (f) 705 nm.

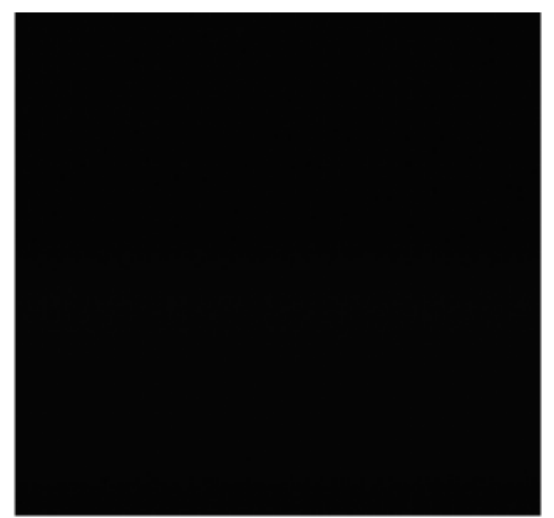

Figura 7. Calibración del negro, nivel basal de la cámara.

\section{a). Sistema en configuración con filtros interferen-} ciales:

Con este sistema (figura 8) se obtuvo un grupo de imágenes multi-espectrales de una muestra de tejido de la capa interna de la aorta. Las longitudes de onda utilizadas para dicha adquisición fueron las siguientes: 436nm (FWNM 8.94nm), 520nm (FWNM 40nm), 546nm (FWNM 8.3nm), 578nm (FWNM $6.72 \mathrm{~nm}$ ), 650nm (FWNM 40nm) y 705nm (FWNM $239 \mathrm{~nm}$ ). Dichas imágenes fueron procesadas por medio de algoritmos, PCA. A continuación se presentan los resultados obtenidos:

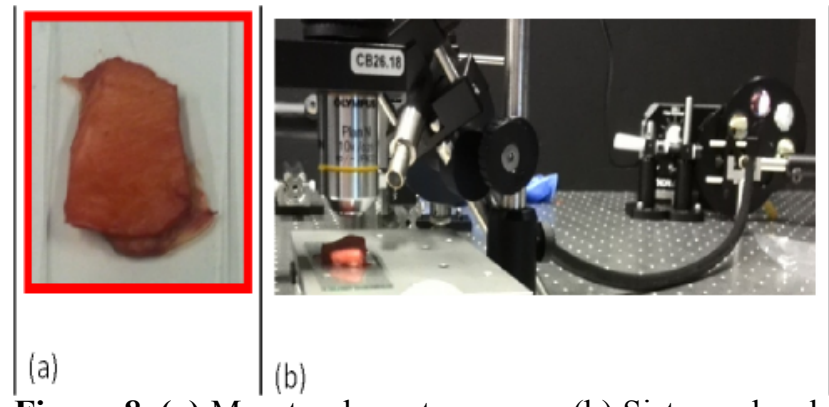

Figura 8. (a) Muestra de aorta vacuna. (b) Sistema de adquisición de imágenes multiespectrales.

a).Mediante PCA, se calcularon 3 componentes principales de las imágenes adquiridas (Figura 9(a) ). Éstas dan cuenta de la microestructura de una sección longitudinal de la aorta, específicamente de la túnica intima, que se encuentra formada principalemete por una capa de células epiteliales aplanadas sostenidas por una gruesa capa de fibras elásticas.
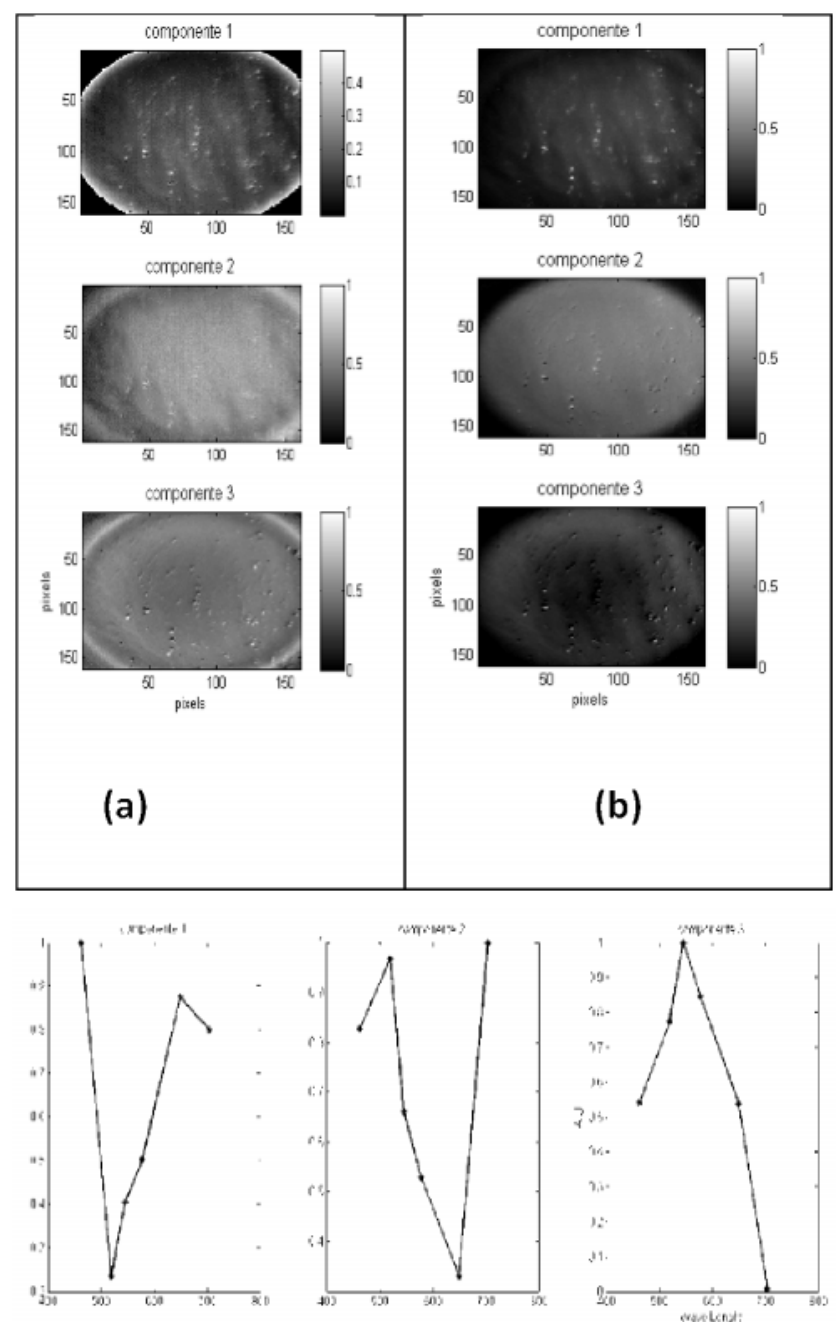

Figura 9. Procesamiento de las imágenes. (a) PCA. (b) ICA. (c) Mapas de concentración. 
b). Empleando el algoritmo ICA, se calcularon tres componentes de la muestra de aorta (figura 9b), junto con sus respectivos mapas de concentracion (figura 9c).

\section{CONCLUSIONES}

A partir del uso de filtros interferenciales, se logró obtener un sistema de adquisición de imágenes multiespectrales de tejido aortico vacuno con calibración espectral. Se implementaron algoritmos de PCA e ICA, donde el primero permitió observar la orientación de las fibras elásticas que son las predominantes en este tejido. En el ICA, dado que el espectro de reflectancia de un tejido biologico puede observarse como la combinacion lineal de la reflectancia de cada uno de los componentes que hacen parte del mismo, la figura $9 \mathrm{c}$ da cuenta de los componentes implicitos en el tejido cardiovascular. Dichos componentes pueden estar relacionados con hemoglobina, lipidos, caroteno, colageno y elastina. Este estudio teórico y práctico aporta en el sentido de lograr un nuevo camino hacia el desarrollo de una herramienta diagnóstica en el área cardiovascular.

\section{AGRADECIMIENTOS}

Los autores agradecen el apoyo económico del Instituto Tecnológico Metropolitano (ITM) y la Universidad Pontificia Bolivariana de Medellín (UPB), mediante el proyecto de investigación con código P14129.

\section{BIBLIOGRAFÍA}

1. Hardeberg, J. Y., Schmitt, F., Brettel, H., Crettez, J. P., \& Maitre, H. (1999). Multispectral image acquisition and simulation of illuminant changes. Colour imaging: vision and technology, 145164.

2. Jolivot, R., Vabres, P., \& Marzani, F. (2011). Reconstruction of hyperspectral cutaneous data from an artificial neural network-based multispectral imaging system. Computerized medical imaging and graphics, 35(2), 85-88.

3. Nakappan, S., Park, S. Y., Serachitopol, D., Price, R., Cardeno, M., Au, S., \& Pikkula, B. M. (2007). Methodology of a real-time quality control for the multispectral digital colposcope (MDC). Gynecologic oncology, 107(1), S215-S222.

4. Orfanoudaki, I. M., Themelis, G. C., Sifakis, S. K., Fragouli, D. H., Panayiotides, J. G., Vazgiouraki, E. M., \& Koumantakis, E. E. (2005). A clinical study of optical biopsy of the uterine cervix using a multispectral imaging system. Gynecologic oncology, 96(1), 119-131.

5. Wieringa, F. P., Mastik, F., Duncker, D. J. G. M., Bogers, A. J. J. C., Zeelenberg, C., \& Van Der Steen, A. F. W. (2006, September). Remote optical stereoscopic multispectral imaging during cardiac surgery. In Computers in Cardiology, 2006 (pp. 693-696). IEEE.

6. Park, J., Pande, P., Shrestha, S., Clubb, F., Applegate, B. E., \& Jo, J. A. (2012). Biochemical characterization of atherosclerotic plaques by endogenous multispectral fluorescence lifetime imaging microscopy. Atherosclerosis, 220(2), 394-401.

7. Mansouri, A., Hardeberg, J. Y., Gouton, P., \& Marzani, F. S. (2005). Optical calibration of a multispectral imaging system based on interference filters. Optical Engineering, 44(2), 027004-027004.

8. Hardeberg, J. Y., Schmitt, F., \& Brettel, H. (2002). Multispectral color image capture using a liquid crystal tunable filter. Optical engineering, 41(10), 2532-2548.

9. Dicker, D. T., Lerner, J., Van Belle, P., Barth, S. F., Herlyn, M., Elder, D. E., \& El-Deiry, W. S. (2006). Research Paper Differentiation of Normal Skin and Melanoma using High Resolution Hyperspectral Imaging. Cancer biology \& therapy, 5(8), 1033-1038.

10. Duque, D., \& Garzón, J. (2013). Effects of both diffractive element and fiber optic based detector in a chromatic confocal system. Optics \& Laser Technology, 50, 182-189.

11. Larsen, E. L., Randeberg, L. L., Olstad, E., Haugen, O. A., Aksnes, A., \& Svaasand, L. O. (2011). Hyperspectral imaging of atherosclerotic plaques in vitro. Journal of biomedical optics, 16(2), 026011026011 . 